\title{
Lobar Pneumonia in a Farmer Responded to Anti-melioidosis Antibiotics
}

Chee Yik Chang

Department of Medical, Sarawak General Hospital, Kuching, Malaysia.

Corresponding Author

Chee Yik Chang

Mobile:

$+60182856630$

E mail:

ccyik28@gmail.com

Key words: Lobar

Pneumonia;

Farmer;melioidosis; Antibiotics
A 46-year-old male, farmer, with no prior medical illness presented with a onemonth history of intermittent right-sided pleuritic chest pain and productive cough. $\mathrm{He}$ complained of fever and breathlessness 3 days before current presentation to the district hospital. $\mathrm{He}$ denied any haemoptysis, night sweats, or contact with pulmonary tuberculosis. On examination, the vital signs were normal and lung auscultation revealed bronchial breath sounds and crepitation over the right upper zone. Chest radiograph demonstrated a right upper lobe consolidation with presence of air bronchogram and abdominal ultrasonography was normal. He was admitted for intravenous antibiotic therapy for a presumptive diagnosis of community-acquired pneumonia. He was initially treated with intravenous amoxicillin-clavulanic acid $1.2 \mathrm{~g}$ every 8 hours. Differential diagnosis of pulmonary tuberculosis was also considered but later ruled out as the sputum for direct smear microscopy, GenXpert and culture for Mycobacterium tuberculosis were negative. The melioidosis IgM (ELISA), multiple blood and sputum cultures were also negative.
A PCR-based confirmatory test for melioidosis was however not available at our setting. In the ward, he continued to have persistent fever and repeated chest radiograph after 1 week of antibiotic showed worsening of right upper lobe consolidation. At this point in time, melioidosis was suspected due to the risk factor of farming. Antibiotic was then changed to intravenous ceftazidime $2 \mathrm{~g}$ every 6 hours. The patient responded positively as evidenced by the resolution of fever and respiratory symptoms, and repeated chest radiograph showed improving consolidation at 2 weeks. $\mathrm{He}$ was discharged after completing a 2-week course of intravenous ceftazidime, and simultaneously started on oral trimethoprim-sulfamethoxazole as eradication therapy. Upon review in the outpatient clinic 6 weeks later, chest radiograph showed marked improvement with minimal residual right upper lobe consolidation. He was clinically well and did not show any symptoms of relapse. He was planned for eradication therapy of 20 weeks' duration.
A 46-year-old male, farmer, with no prior medical illness presented with a one-month history of intermittent rightsided pleuritic chest pain and productive cough. $\mathrm{He}$ complained of fever and breathlessness 3 days before current presentation to the district hospital. He denied any haemoptysis, night sweats, or contact with pulmonary tuberculosis. On examination, the vital signs were normal and lung auscultation revealed bronchial breath sounds and crepitation over the right upper zone. Chest radiograph demonstrated a right upper lobe consolidation with presence of air bronchogram (figure 1a), and abdominal ultrasonography was normal. He was admitted for intravenous antibiotic therapy for a presumptive diagnosis of community-acquired

pneumonia. He was initially treated with intravenous amoxicillin-clavulanic acid $1.2 \mathrm{~g}$ every 8 hours. Differential diagnosis of pulmonary tuberculosis was also considered but later ruled out as the sputum for direct smear 
microscopy, GenXpert and culture for Mycobacterium tuberculosis were negative. The melioidosis IgM (ELISA), multiple blood and sputum cultures were also negative. A PCR-based confirmatory test for melioidosis was however not available at our setting. In the ward, he continued to have persistent fever and repeated chest radiograph after 1 week of antibiotic showed worsening of right upper lobe consolidation (figure 1b). At this point in time, melioidosis was suspected due to the risk factor of farming. Antibiotic was then changed to intravenous ceftazidime $2 \mathrm{~g}$ every 6 hours.
The patient responded positively as evidenced by the resolution of fever and respiratory symptoms, and repeated chest radiograph showed improving consolidation at 2 weeks (figure 1c). He was discharged after completing a 2-week course of intravenous ceftazidime, and simultaneously started on oral trimethoprim-sulfamethoxazole as eradication therapy. Upon review in the outpatient clinic 6 weeks later, chest radiograph showed marked improvement with minimal residual right upper lobe consolidation (figure $1 \mathrm{~d}$ ). $\mathrm{He}$ was clinically well and did not show any symptoms of relapse. He was planned for eradication therapy of 20 weeks' duration. 


\section{Figure1a}

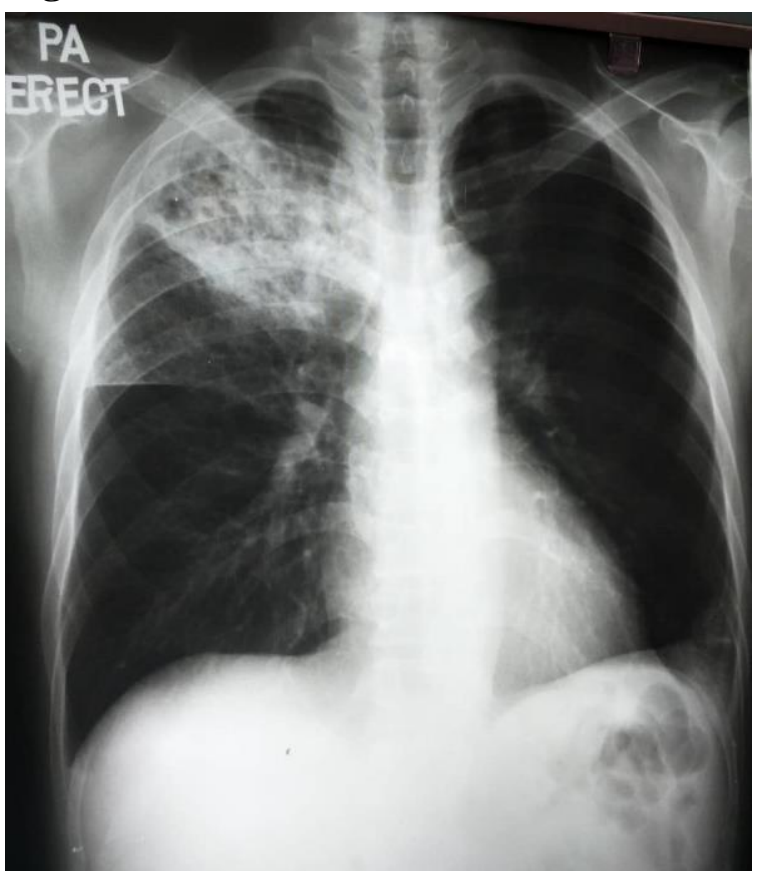

\section{Figure1c}

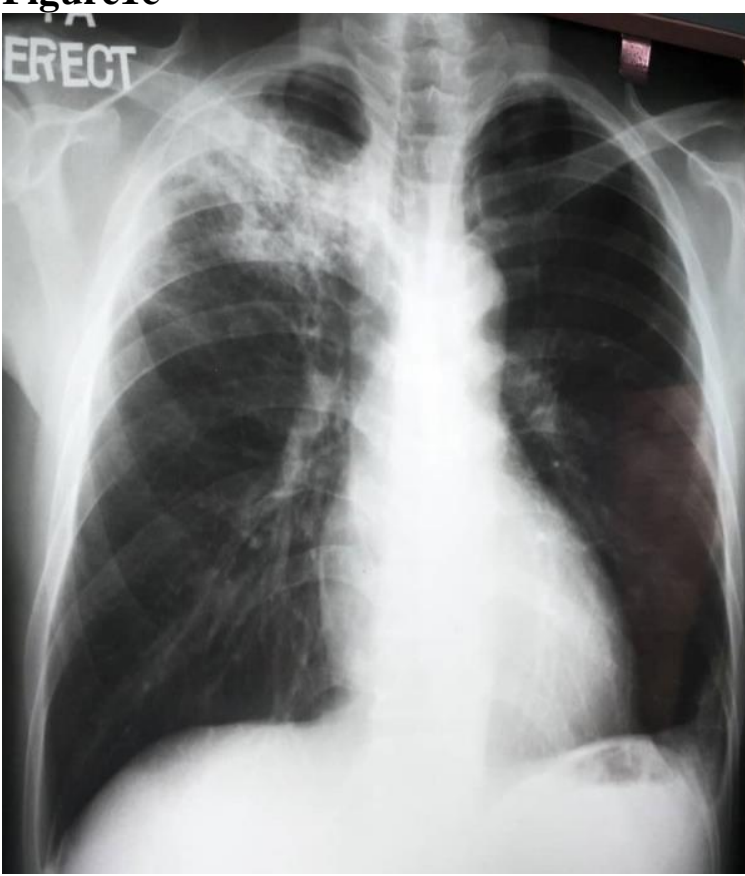

\section{Figure1b}

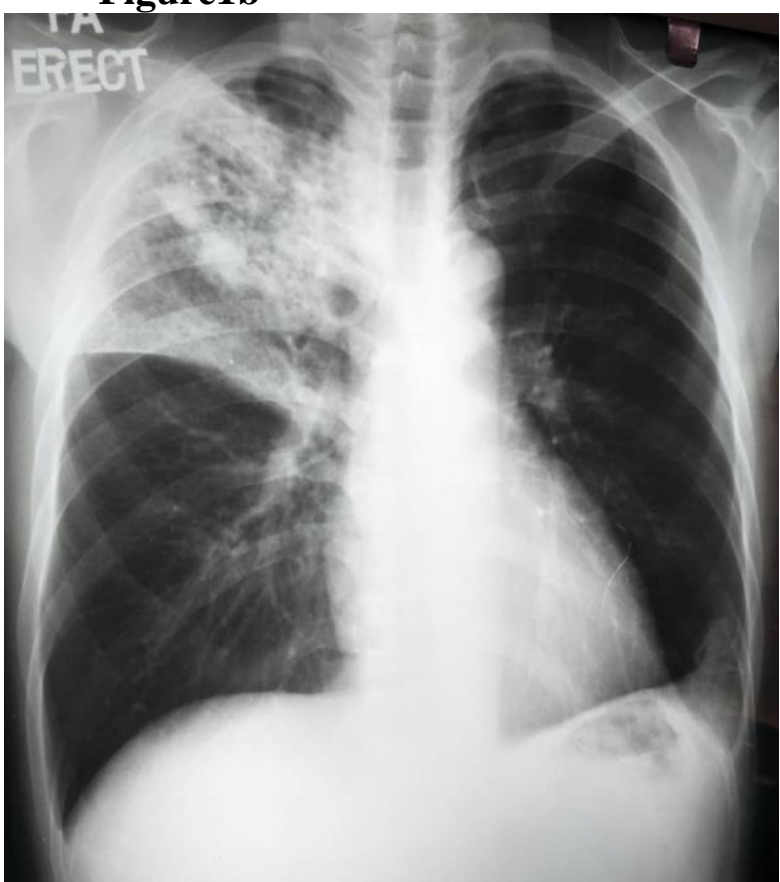

Figure1d

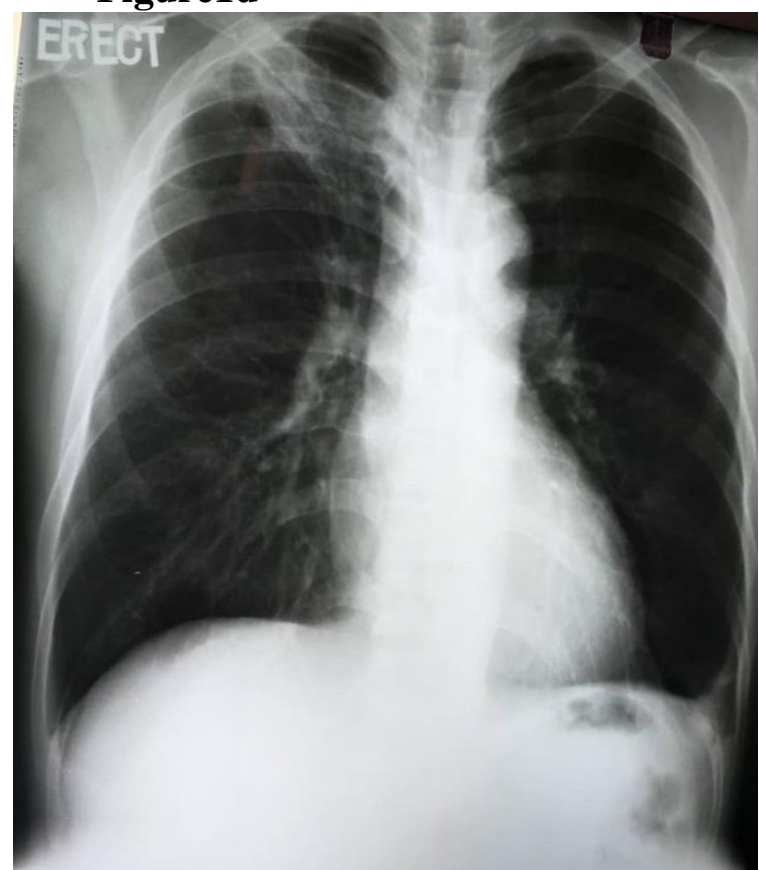

Figure 1 shows the serial progression of chest radiograph. (a) on admission, (b) after 1-week of amoxicillin-clavulanic acid, (c) after completion of ceftazidime for 2 weeks, (d) on $6^{\text {th }}$ week trimethoprim-sulfamethoxazole therapy.

Melioidosis is an infectious disease caused by Burkholderia pseudomallei, and can present with a wide range of clinical manifestations, including pneumonia, internal organ abscesses, neurological melioidosis, skin and soft tissue/joint infection, and bacteraemia [1]. In pulmonary melioidosis, the upper lobe is commonly involved with formation of early 
cavitation which can progress rapidly. The radiographic features of melioidosis sometimes mimicked pulmonary tuberculosis with upper lobe involvement in the subacute or chronic forms [2]. In rare instances, an air crescent sign can also be seen in pulmonary melioidosis [3]. This case poses a diagnostic challenge because both the cultures and serology test were negative for $B$. pseudomallei but the patient showed marked clinical and radiological improvement with melioidosis treatment. Although isolation of B. pseudomallei from body fluids of patients is the "gold standard" in the diagnosis of melioidosis, a negative culture does not exclude melioidosis as culture has low sensitivity for diagnosis of melioidosis [4]. Melioidosis should be suspected in patients presenting with fever who have a history of residency in, or travel to a area where melioidosis is endemic; an occupation with exposure to soil or water that might contain B. pseudomallei; and the presence of risk factors such as diabetes mellitus or kidney disease [5]. Diagnosis of melioidosis in a resource-limited setting is extremely challenging due to lack of more sophisticated test, and the outcome is greatly dependent on the clinicians' experience and competence.
Ethical considerations: Written informed consent was obtained from the patient for publication of this case report.

\section{Funding: None}

Conflict of interest: The author declares that he has no conflict of interest.

\section{REFERENCES}

1. Cheng AC, Currie BJ. Melioidosis: Epidemiology, pathophysiology, and management. Clin Microbiol Rev 2005; az18(2):383-416.

2. Reechaipichitkul W. Clinical manifestation of pulmonary melioidosis in adults. Southeast Asian J Trop Med Public Health 2004; 35(3):664-669.

3. Chang CY. A case report of pulmonary melioidosis with the air crescent sign. Radiol Infect Dis. 2020;7(1):31-34.

4. Limmathurotsakul D, Jamsen K, Arayawichanont A, Simpson JA, White LJ, Lee SJ, et al. Defining the true sensitivity of culture for the diagnosis of melioidosis using Bayesian latent class models. PLoS One 2010; 5(8):e12485.

5. Limmathurotsakul D, Peacock SJ. Melioidosis: a clinical overview. Br Med Bull 2011;99:125-39. 